\title{
Endogenous RNA editing of a nuclear gene BOSS triggers flowering in tomato
}

Wenqian Wang ${ }^{\#, 1,2}$, Jie Ye $\mathrm{Y}^{\#, 1}$, Chuying $\mathrm{Yu}^{1}$, Qingmin $\mathrm{Xie}^{1}$, Xin Wang ${ }^{1}$, Huiyang Yu ${ }^{1}$, Jianwen

Song ${ }^{1}$, Changxing $\mathrm{Li}^{1}$, Long Cui ${ }^{1}$, Heyou Han ${ }^{2}$, Changxian Yang ${ }^{1}$, Hanxia $\mathrm{Li}^{1}$, Yongen $\mathrm{Lu}^{1}$, Taotao

Wang ${ }^{1}$, Yuyang Zhang ${ }^{1}$, Junhong Zhang ${ }^{1}$, Bo Ouyang ${ }^{1}$, Zhibiao Ye*,1

1. Key Laboratory of Horticultural Plant Biology, Ministry of Education, Huazhong Agricultural University, Wuhan 430070, China.

2. College of Food Science and Technology, Huazhong Agricultural University, Wuhan 430070, China.

*Corresponding Author, E-mail: zbye@mail.hzau.edu.cn (Z.Y.).

\# These authors contributed equally: Wenqian Wang (W.W.), Jie Ye (J.Y.). 


\begin{abstract}
RNA editing is defined as the production of transcripts with RNA sequences different from those of the DNA template. Most of previous RNA editing studies have focused on organelles, while RNA editing of nuclear transcripts is largely unknown. Here, we describe the first example of nuclear transcript RNA editing in plant, the BOSS RNA editing regulates tomato flowering. The SNP (SNP1) located in the 5'UTR of BOSS gene (the Balancer of $\underline{S} P$ and $\underline{S} F T$ ) is associated with tomato early flowering, and two transcripts of BOSS produced by SNP1 associated RNA editing show functional differentiation, where BOSS- $\beta$ transcript promotes flowering while $B O S S$ - $\alpha$ does not. Furthermore, these two transcripts of $B O S S$ are shown to regulate $S P$ (anti-florigen) pathway at transcription level in the shoot apical meristem (SAM). Our findings reveal a new layer of complexity in the control of plant stem cell proliferation and provide the evidence of RNA-editing of a single gene for flowering, suggesting that molecular breeding programs to increase the RNA-editing efficiency may improve the productivity of tomatoes and other agricultural organisms.
\end{abstract}




\section{INTRODUCTION}

Crop yields are strongly associated with flowering time, and for many plants, the significance of flowering mainly involves two aspects: (i) balancing and optimizing their market supply for human demand, and (ii) enhancing their adaptability and expanding the geographical range of cultivation (Andres and Coupland, 2012; Wang et al., 2016). Tomato (Solanum lycopersicum), a main vegetable crop originating in the Andes of South America with a worldwide distribution, is classified as a day-neutral and temperature-loving plant, and its flowering and fruiting can be restricted under adverse conditions (Calvert, 1959; Song et al., 2013; Soyk et al., 2017).

Flowering is a sign for the transition of plants from vegetative to reproductive growth, and this process is precisely regulated by both genetic factors and external environmental factors (Imaizumi and Kay, 2006; Jung and Muller, 2009; He, 2012). In the long-day plant Arabidopsis, flowering has been shown to be controlled by five pathways, including vernalization, photoperiod, gibberellin, autonomous and senescence pathways (Srikanth and Schmid, 2011). These five pathways were integrated into a network through three central genes: CO (CONSTANS) (Suarez-Lopez et al., 2001; Valverde et al., 2004), FT (FLOWERING LOCUS T) (Kardailsky et al., 1999; Tiwari et al., 2010), and FLC (FLOWERING LOCUS C) (Michaels and Amasino, 1999; Searle et al., 2006). In the short-day plant rice, several genes have been identified to control the flowering time, such as Hd3a (Heading date3a) and its paralog RFT1 (RICE FLOWERING LOCUS T1) encoding florigens (Kojima et al., 2002; Komiya et al., 2008; Komiya et al., 2009).

Despite increasing reports of single genes responsible for the variation of important traits like plant flowering (Teo et al., 2014), little is known about the synergistic regulation of flowering by different flowering genes or one gene through different transcriptional or post-transcriptional modification. In Arabidopsis, FLM- $\beta$ and FLM- $\delta$, two FLM protein splice variants with opposite functions, are shown to compete for interaction with the floral repressor SVP to dynamically regulate flowering at different temperatures (Pose et al., 2013). In sympodial plants such as tomato (Solanum lycopersicum) (Knapp et al., 2004; MacAlister et al., 2012), the differentiation of the first floral meristem occurs after the vegetative growth of several leaves, which determines the flowering time of tomato, termed as the first inflorescence node (FIN), followed by the vegetative 
growth from the axil of the youngest leaf through a sympodial vegetative meristem (SYM) to three leaves and then transition into a flower (MacAlister et al., 2012). Sympodial cycling is regulated by the balance between flower-promoting (florigen) and flower-repressing (anti-florigen) activities in the shoot apical meristem (SAM), with $S P$ as a flowering repressor to transform tomato indeterminate shoot architecture into determinate vines (Pnueli et al., 1998), while SFT as a genetic originator of the flowering hormone florigen to promote flowering (Lifschitz et al., 2006). The dose-sensitivity balance of $S F T$ and $S P$ transcripts in SAM determines their differentiation, which further influences inflorescence architecture and increases yield by producing more inflorescence (Jiang et al., 2013). However, the detailed molecular mechanism underlying the dose effect between $S P$ and $S F T$ remains unknown.

As a post-transcriptional processing event, RNA editing is defined as an RNA product different from its DNA template, which is a supplement to the central dogma (the flow of genetic information from DNA to RNA to protein). RNA editing was first discovered in Trypanosoma brucei mitochondria (Benne et al., 1986). In plants, RNA editing (the C-to-U transition) is highly prevalent and restricted within organelles (mitochondria and chloroplasts), which can alter the coding sequences of the organellar transcripts (Rajasekhar and Mulligan, 1993; Gerke et al., 2020; Small et al., 2020). Thus far, despite reports on the genome-wide RNA editing of nuclear transcripts in humans (Chen, 2013) and Arabidopsis (Meng et al., 2010), none of these predictions has been verified by gold-standard Sanger sequencing, and few studies have been performed on the involvement of nuclear transcript RNA editing in regulating important traits, such as plant flowering.

In this study, we report the identification of BOSS (Balancer of $\underline{S} P$ and $\underline{S} F T$ ) as the causal gene corresponding to our previously identified FIN-related QTL (Ye et al., 2020), qFIN9 (QTL of FIN on chromosome 9) in tomato (Table S1). BOSS has two transcripts (BOSS- $\alpha$ and BOSS- $\beta$ ) attributed to endogenous RNA editing. BOSS- $\beta$ rather than BOSS- $\alpha$ is shown to promote flowering because of their different regulatory patterns with $S P$. This study reveals a dynamic mechanism in floral initiation which confers to the balance of $S P$ and $S F T$ in tomato. 


\section{RESULTS}

\section{Characterization of Endogenous RNA editing of $B O S S$}

An E3 ubiquitin-protein ligase (Solyc09g010650.1), which has been reported to regulate flowering in rice (Yang et al., 2015), was preferred as the candidate of $q F I N 9$. BOSS contains only one single exon and encodes a 176-amino acid protein with a molecular mass of $16.74 \mathrm{kDa}$, with an E3 ubiquitin-protein ligase domain present in its C-terminal region (amino acids 78-120) and a transmembrane domain located in its N-terminal region (amino acids 7-26) (Fig. S1A). Sequence analysis showed that the orthologous gene of BOSS in Arabidopsis thaliana is an ATL33 gene, which lacks relevant research, with almost all homologous genes present in monocotyledons (Fig. $\mathrm{S1B}$ ). The expression of BOSS is high in seed and opened flower, but low in root and bud (Fig. S1C). Only one polymorphism, Chr09_3978372 (A/T, SNP1) in the 5 'UTR of BOSS, and no significant difference was detected in the BOSS expression between the Low-FIN and High-FIN lines (Fig. S2), suggesting that the two BOSS haplotypes are independent of the transcriptional level.

Interestingly, in the individual plants of TS54 $\left(B O S S^{\mathrm{AA}}\right)$, we found two transcripts (BOSS- $\alpha$ and $B O S S-\beta)$ were distinguished by a SNP (SNP2, C/U at position 457 of RNA sequence, with amino acid being changed from $\mathrm{R}$ to $\mathrm{W}$ at position 153 of amino acid sequence) (Fig. 1A). As we know, RNA editing has been defined as a single-base difference between DNA and RNA, and the common model of RNA editing in plant organelles is "C>U", indicating a good match of the phenomenon we found in the nuclear gene BOSS for RNA editing. Random primers were used to detect the RNA editing of organelles previously (Wang et al., 2019), but BOSS is a nuclear gene decorated with a polyA after transcription. Whether the type of reverse transcription will influence the detection of RNA editing was investigated by using three methods for reverse transcription: only random primers, only oligodT primers, and a combination of the two. The random \& oligodT primers were shown to detect the RNA editing of BOSS more stably and thus were used in further studies (Fig. S3). To verify the RNA editing in BOSS, we sequenced the gDNA and cDNA of BOSS in the three individuals of TS54 belonging to BOSS $\mathrm{AA}$. In Fig.1B, the gDNA sequence of BOSS was shown as $\mathrm{C}$ at position 457 in TS54, in contrast to the heterozygous peak of $\mathrm{C}$ and $\mathrm{T}$ for 
the cDNA sequence at this position.

The tissue distribution of RNA-editing in BOSS was analyzed by using TS54 $\left(B O S S^{\mathrm{AA}}\right)$ as an example, which was found to be widely present in the root, stem tip, leaf, cotyledon, flower, bud, seed, and fruit, with the RNA-editing efficiency being highest in the stem tip and lowest in the fruit (Fig.1C). As tomato flowering is strictly regulated by light, we investigated its effect on the RNA-editing efficiency of BOSS in cotyledon, stem, leaf and stem tip (Fig.1D). Under light treatment, the RNA-editing efficiency was continuously induced and reached its peak at $24 \mathrm{~h}$ in stem shoot, most rapidly induced at $4 \mathrm{~h}$ and followed a downward trend in cotyledon, and exhibited a decrease first and then an increase to the peak at $20 \mathrm{~h}$ in leaf and stem. In terms of time, RNA-editing efficiency reached its peak most rapidly (at $4 \mathrm{~h}$ ) in cotyledon (an increase of $8 \%$ per hour), 16 and 20 hours earlier than in leaf and stem tip (an increase of 1\% per hour), respectively. The results of the light experiments suggest that the earliest receptor organ for the photoinduced RNA-editing efficiency of BOSS was cotyledon, followed by leaf, stem and stem tip.

The 3D structure prediction of the BOSS protein showed that RNA-editing leads to the production of BOSS genes with different protein structures (Fig. 1E). Moreover, sequence analysis revealed more predicted protein binding sites for BOSS- $\beta$ than BOSS- $\alpha$, suggesting the higher activity of the former (Fig. S4A). The similarities and differences between BOSS- $\alpha$ and BOSS- $\beta$ in the subcellular localization were investigated by creating BOSS-YFP fusion proteins, which were transiently expressed in Nicotiana benthamiana. The fluorescent signals of YFP were seen to overlap with those of N-RFP (a marker for the nucleus) and M-RFP (a marker for the plasma membrane), suggesting that both BOSS- $\alpha$ and BOSS- $\beta$ are located in the nuclear and plasma membranes (Fig. S4B, C).

The RNA editing in BOSS and its association with SNP1 were verified by sequencing the gDNA and cDNA of BOSS in 23 accessions, with 12 of them representing $B O S S^{\mathrm{TT}}$ and 11 of them belonging to $B O S S^{\mathrm{AA}}$. In Fig.1F, BOSS ${ }^{\mathrm{AA}}$ was seen to have higher RNA-editing efficiency (an average of $43 \%$ ) than $B O S S^{\mathrm{TT}}$ (an average of $8.9 \%$ ), revealing the association of RNA-editing efficiency with SNP1 in 5 'UTR.

Whether RNA-editing of BOSS is conserved in different lineages was tested by identifying the BOSS orthologous proteins in potato (Solanum tuberosum) and melon (Cucumis melo), of which more RNA editing types were found than tomato (Fig. 1G). The above results suggest that 
RNA editing of different complexity may be conserved in different flowering plants.

\section{$B O S S-\beta$ instead of $B O S S-\alpha$ promotes flowering in tomato}

The functional differences between BOSS- $\alpha$ and BOSS- $\beta$ in tomato flowering were evaluated by generating two overexpression constructs containing allele BOSS- $\alpha$ and BOSS- $\beta$ and introducing them into TS221 (BOSS ${ }^{\mathrm{TT}}$ with low RNA-editing) and ZY3 (BOSS ${ }^{\mathrm{AA}}$ with high RNA-editing), respectively. The BOSS RNA levels showed a significant increase in the three independent BOSS- $\beta$ overexpression lines in $\mathrm{T}_{1}$ generation (Fig. $2 \mathrm{~A}$ ), with the BOSS- $\beta$ transcript being $100 \%$ in the BOSS- $\beta$ overexpression lines (Fig. 2B). Compared with TS221, the three BOSS- $\beta$ overexpression lines had lower FIN and FSIN (first to second inflorescence node) (Fig. 2 C-E). The early flowering of BOSS- $\beta$ overexpression lines was further evaluated by microscopic observation of paraffin sections and SAM scanning electron micrographs from wild-type tomato and BOSS- $\beta$ overexpression lines (Fig. 2F, G). The FM (flower meristem) of the primary meristem was observed to occur earlier in the BOSS- $\beta$ overexpression lines than in the wild-type tomato, leading to early flowering. Moreover, the lower FIN and FSIN increased the flower trusses, thereby increasing the yield per plant in the BOSS- $\beta$ overexpression lines (Fig. 2H, I). Interestingly, the overexpression of BOSS- $\beta$ led to the self-pruning of a few transgenic plants (Fig. S5), suggesting that BOSS may regulate self-pruning by some dosage interaction with other related genes. Conversely, the overexpression of BOSS- $\alpha$ failed to affect the differentiation of FIN, FSIN or FM in ZY3 (Fig. S6). The results of the transgenic experiments strongly suggest that BOSS- $\beta$ rather than BOSS- $\alpha$ functions as a positive regulator of tomato flowering.

\section{BOSS $-\beta$ promoting flowering by inhibiting the expression of SP}

How different BOSS transcripts regulate tomato flowering was explored by RNA-seq analysis of ZY3/BOSS- $\alpha-\mathrm{OE}$ and TS221/BOSS- $\beta$-OE lines. Differential gene expression analysis identified the up- and down-regulation of 36 and 33 genes, respectively, in TS221/BOSS- $\beta$-OE lines (fold change $>2$; FDR $<0.05$ ), in contrast to the up- and down-regulation of only 7 and 2 genes, respectively, in ZY3/BOSS- $\alpha-\mathrm{OE}$ line (Table S2, Fig. S7A, B). Additionally, GO enrichment analysis of the upregulated genes in TS221/BOSS- $\beta$-OE reported 'MADS-box transcription factor' 
as the top term and included the known flowering-related gene FLC-like (Solyc12g087810) (Fig. S7C). The upregulation of these three MADS-box transcription factors (Solyc12g087810, Solyc12g087820, Solyc12g087830) was verified by quantitative RT-PCR (RT-qPCR) (Fig. S7D-F).

Moreover, to analyze the dynamic expression changes of flowering related genes in BOSS over-expression lines, we performed quantitative RT-PCR (qRT-PCR) at the different stages of seedling development (Fig. 3). In agreement with the early flowering phenotype, we found that $B O S S-\beta$ inhibited the expression of anti-florigen $S P$ (-7.5 folds) at $5^{\text {th }}$ leaf stage to promote flowering, whereas the expression of $S P$ (-8.6 folds) was promoted at $6^{\text {th }}$ leaf stage in $B O S S-\alpha$ over-expression line (Fig. 3A). The expression of florigen SFT was not significantly affected in both BOSS- $\alpha$ and $-\beta$ over-expression lines (Fig. 3B). These results indicated that BOSS- $\beta$ regulated flowering of tomato by regulating $S P$ expression.

\section{DISCUSSION}

The genome editing events of plant organelles are mainly investigated by bioinformatics and experimental approaches (Tang and Luo, 2018; Yan et al., 2018; Small et al., 2020), while the RNA editing of nuclear transcripts is only studied using bioinformatics predictions in plant (Meng et al., 2010). In this study, we identified the RNA editing of a nuclear gene BOSS by Sanger sequencing method which was considered as the gold standard for identification of RNA editing (Zhao et al., 2019). Interestingly, except for TS-225(C/T heterozygosity), all the re-sequenced accessions showed $\mathrm{C} / \mathrm{C}$ homozygou of the RNA-editing site at DNA level (https://solgenomics.net/organism/Solanum lycopersicum/tomato 360), suggesting that the variation of this site in natural population may trend to transit from DNA level to RNA level.

Except for tomato, the RNA-editing also widely identified in other species, such as potato and melon (Fig. 1G). Like RNA-editing of BOSS regulate $S P$ in tomato, the more extensive editing types of BOSS orthologous in potato may be of great significance for tuber development which was regulated by StSP6A, the orthologous of tomato SP (Abelenda et al., 2014). And the significance seems to be greater due to a dual editing site of BOSS orthologous gene in melon (Fig. 
$1 \mathrm{G)}$.

In summary, our results confirm the existence of RNA editing of the nuclear transcript BOSS (Fig 1). Additionally, we constructed a sophisticated regulatory pathway that the natural variation of SNP1 located in the palindromic sequence of BOSS 5 'UTR leads to different RNA-editing efficiency, which in turn affects the transcripts of BOSS- $\alpha$ and $-\beta$. Finally, different transcripts of $B O S S$ regulate tomato flowering by affecting the expression level of $S P$ at seedling stage, BOSS- $\beta$ decreased the expression of $S P$ but $B O S S-\alpha$ increased the expression of $S P$ (Fig. 4). Although the expression of $S P$ both altered in BOSS- $\alpha$ and $-\beta$ overexpression lines, the change expression phase, vital to stem apical meristem fate determinate, was different $\left(5^{\text {th }}\right.$ leaf stage for BOSS- $\beta$ and $6^{\text {th }}$ leaf stage for BOSS- $\alpha$ ) (MacAlister et al., 2012). The new knowledge about RNA-editing of tomato flowering will facilitate genome-wide research on the regulatory role of RNA editing in various biological processes.

\section{METHODS}

\section{Phylogenetic analysis of $B O S S$ gene family}

The amino acid sequences of BOSS and its homologues were aligned using the CLUSTALX (version 2.1) software. The neighbor-joining tree was constructed using aligned full-length amino acid sequences (MEGA6), with bootstrap values from 1,000 replicates indicated at each node. Bar $=0.1$ amino acid substitutions per site. The tree was visualized by the online tool Interactive Tree of Life (iTOL).

\section{Light treatment}

TS54 plants (high RNA-editing efficiency) were grown in plastic pots in the greenhouse under a 16-h light and 8-h dark at $25^{\circ} \mathrm{C}$. At the seedling age of three leaves and one hear, the plants were treated with continuous light and samples of different tissues were collected at $0 \mathrm{~h}, 4 \mathrm{~h}, 8 \mathrm{~h}, 12 \mathrm{~h}$, $16 \mathrm{~h}, 20 \mathrm{~h}, 24 \mathrm{~h}$ and $48 \mathrm{~h}$ post light treatment to determine the RNA-editing efficiency of BOSS in different tomato tissues. 


\section{RNA isolation and gene expression analysis}

To analyze the dynamic expression changes of $S P / S F T$ at different developmental stages, tomato seedlings were planted and cultured as above, and shoot tip tissues were collected from 3th leaf stage to 7th leaf stage, respectively. Total RNA was isolated from tomato using TRIZOL reagent (Invitrogen, USA) according to the manufacturer's instructions. The cDNAs were synthesized from $1 \mu \mathrm{g}$ total RNA using HiScript ${ }^{\circledR} I I$ Reverse Transcriptase (Vazyme, China) following the manufacturer's protocol. Gene expression was investigated by quantitative real-time PCR (qRT-PCR) as previously described (Ye et al., 2019). The actin gene (Solyc11g008430) was used as an internal standard and the relative expression of specific genes was measured using the cycle threshold (Ct) $2^{-\Delta \Delta C t}$ method.

\section{RNA-Seq analysis}

At the seedling age of six weeks, the shoot tip tissues from TS221, BOSS- $\alpha-\mathrm{OE}$ and BOSS- $\beta-\mathrm{OE}$ were frozen in liquid nitrogen and kept at $-80^{\circ} \mathrm{C}$ until further use. An RNA sample was obtained from three plants of each genotype, with three biological replicates $(3 \times 3=9$ plants $)$ for each genotype. Total RNAs were extracted using the same method as described above and then sent to the Berry Genomics Company (Beijing, China), where the libraries were constructed and sequenced using single-ended sequencing of Illumina Novaseq6000. The sequencing data can be accessed at the Sequence Read Archive (SRA ID: PRJNA673993). The reference genome sequences (SL2.50 version) of S. lycopersicum were downloaded from the SOL Genomics Network database (http://solgenomics.net/organism/Solanum_lycopersicum/genome). Here, genes with a $P$ value $<0.01$ and a $\log _{2}$ ratio $>1.0$ or $<0.5$ were considered differentially expressed. Sequence analysis and differential expression analysis were performed with a method provided by Novogene. More detailed information is provided on the Novogene website (www.novogene.com).

\section{Analysis of RNA editing efficiency in BOSS}

The coding sequence of BOSS containing RNA editing site was amplified by PCR with cDNA as the templet and the specific primers (Table S3) using Phanta Super-Fidelity DNA Polymerase 
(Vazyme, China). The PCR was conducted as follows: $95{ }^{\circ} \mathrm{C}$ for $3 \mathrm{~min}, 34$ cycles of $95{ }^{\circ} \mathrm{C}$ for $15 \mathrm{~s}$, $56{ }^{\circ} \mathrm{C}$ for $15 \mathrm{~s}, 72{ }^{\circ} \mathrm{C}$ for $30 \mathrm{~s}$, and $72{ }^{\circ} \mathrm{C}$ for $5 \mathrm{~min}$. The PCR products were used as templates for Sanger DNA sequencing (Sequencing was performed by Tianyi Huiyuan Bioscience and Technology, Wuhan, China). The "C" to "T" ( C to U in RNA) editing efficiency was measured by the relative height of the peak of the nucleotide in sequence chromatograms and calculated by the height of "T" / (height of "T" and "C") as previously described (Zhao et al., 2019).

\section{Subcellular analysis}

Using the cDNA of TS221 (low RNA-editing efficiency) and TS54 (high RNA-editing efficiency) as templates, the coding sequences of $B O S S-\alpha$ and $B O S S-\beta$ without the stop codon were amplified by PCR using the primer sequences shown in Table S3, then cloned into the expression vector p101YFP under the control of the CaMV35S promoter by homologous recombination (ClonExpress II One Step Cloning Kit, Vazyme, China). CaMV35S:BOSS- $\alpha / \beta-Y F P$ vector as well as cell nucleus (nucleolus) marker CaMV35S:N-RFP (AtNM, AT4G25630.1) (Degenhardt and Bonham-Smith, 2008), plasmid membrane/nuclear membrane marker CaMV35S:CBLn1-RFP (Batistic et al., 2010) and the control YFP vector (positive control) were transformed into Agrobacterium tumefaciens strain GV3101 and co-infiltrated into leaves of $N$. benthamiana with the suspension as previously described. After 48 -h incubation at $25{ }^{\circ} \mathrm{C}$, the tobacco leaves were used for YFP and RFP observation using Leica Confocal software.

\section{Gene cloning and vector construction}

For the overexpression construct, the $1032 \mathrm{bp}$ fragment containing the 5'UTR (including 8T8A) and CDS region of BOSS was amplified from the genomic DNA of TS221 (9T7 $\mathrm{A}_{5}{ }^{\prime} \mathrm{UTR}^{-} \alpha_{\mathrm{CDS}}$ is the main transcript type) and TS-226 $\left(8 \mathrm{~T} 8 \mathrm{~A}_{5}\right.$ 'UTR $-\beta_{\mathrm{CDS}}$ is the main transcript type) to obtain 9T7A 5'UTR-BOSS- $\alpha_{\mathrm{CDS}}$ and $8 \mathrm{~T} 8 \mathrm{~A}_{5}{ }^{\prime} \mathrm{UTR}-\mathrm{BOSS}-\beta_{\mathrm{CDS}}$ fragments by point mutation. The PCR products were inserted into pDONR221 using the BP enzyme (Invitrogen, USA), and then incorporated into the destination vector pMV3 (driven by the cauliflower mosaic virus (CaMV) 35S promoter) using the LR recombination reaction (Invitrogen, USA). All the recombinant constructs were transformed into the Agrobacterium tumefaciens strain C58 by electroporation and subsequently 
transformed into the tomato genome via explants of cotyledon. To eliminate the influence of $S P$ locus in chromosome 6, the background materials TS221 (SP/SP, 9T7A with lower RNA editing efficiency) and ZY3 (SP/SP, 8T8A with higher RNA editing efficiency) were selected to transform 8T8 A $_{5}{ }^{\prime} \mathrm{UTR}-B O S S-\beta_{\mathrm{CDS}}$ and 9T7A 5'UTR-BOSS- $\alpha_{\mathrm{CDS}}$, respectively.

\section{Data availability}

Data supporting the findings of this work are available within the paper and its Supplementary Information.

\section{Acknowledgments}

This study was supported by the National Natural Science Foundation of China (31991182 and 31872118), the National Key Research and Development Program of China (2017YFD0101902) and CARS-23-A-03.

\section{Author Contributions}

Z.Y., H.L., H.H., J.Z., B.O., W.W., C.Y., X.W., C. Y. and J.Y. designed research and managed the project; W.W., C.Y., Q.X., J.S. and L.C. performed research; W.W., J.Y., H.Y., C.L., Y.L., T.W. and Y.Z. analyzed data; J.Y., W.W. and Z.Y. wrote and modified the manuscript.

\section{Competing interests}

The authors declare no competing financial interests.

\section{Figure Legends}

Fig. 1. RNA editing of BOSS.

(A) Schematic diagram of BOSS RNA editing. BOSS- $\alpha$ with C at position 457 is obtained by 
transcription from gDNA to RNA, and then BOSS- $\beta$ with $\mathrm{U}$ at position 457 is formed by RNA-editing. (B) The RNA editing efficiency of BOSS in TS54. Sequence analysis at RNA-editing site reveals 43\% RNA editing efficiency of BOSS in TS54, with three independent plants used for the analysis. Color traces: G-black, A-green, T-red, C-blue. (C) The RNA-editing efficiency of BOSS in different organs of TS54. (D) The role of RNA-editing efficiency of BOSS in light response. The RNA-editing efficiency of BOSS in different organs of TS54 were detected under 48-h continuous light. (E) Changes in the three-position structure of BOSS protein due to non-synonymous mutations induced by RNA-editing. (F) The RNA-editing efficiency of BOSS in the leaves of two BOSS haplotypes based on SNP1 (A/T). (G) The orthologous genes PGSC0003DMT400023038 and Csa_3g734330 of the BOSS gene in potato and melon showed more RNA editing sites than the tomato BOSS gene. ${ }^{*} P<0.05,{ }^{* *} P<0.01$.

\section{Fig. 2. Elevated BOSS $-\beta$ levels contribute to early flowering in tomato.}

(A) The relative expression of BOSS in BOSS- $\beta$ overexpression transgenic tomato lines. (B) Sequence analysis at RNA-editing site reveals $100 \%$ RNA editing efficiency of BOSS- $\beta$ overexpression versus $6 \%$ in TS221, with three independent plants used for the analysis. Color traces: G-black, A-green, T-red, C-blue. (C) Primary inflorescences (red arrowheads) and side branches (yellow arrowheads) from TS221 (left) and BOSS- $\beta$ overexpression line (right) with early flowering. (D, E) Quantification and comparison of FIN (first inflorescence node) (D) and FSIN (node between first and second inflorescence) (E) in TS221 and BOSS- $\beta$ overexpression lines. (F, G) Stereomicroscopic (F) and scanning electron microscopic $(\mathbf{G})$ analysis of the primary shoot apical meristems from TS221 and BOSS- $\beta$ overexpression lines. (H, I) Quantification (H) 
and images (I) of yields from TS221 and BOSS- $\beta$ overexpression lines. Scale bars: $5 \mathrm{~cm}(\mathrm{C}), 100$ $\mu \mathrm{m}(\mathrm{F}), 100 \mu \mathrm{m}(\mathrm{G})$ and $5 \mathrm{~cm}(\mathrm{I})$. All data in the graphs represent means $\pm \mathrm{SD}(n=3) ;{ }^{*} P<0.05$, $* * P<0.01$

Fig. 3. Relative expression dynamics for $S P$ and $S F T$ in stem apical at the different stages of seedling development.

(A) Relative expression dynamics of $S P$ in stem apical at the different stages of seedling development. $3 \mathrm{~L}-7 \mathrm{~L}$ means from $3^{\text {th }}$ leaf state to $7^{\text {th }}$ leaf state. At $5^{\text {th }}$ leaf stage, compared with WT, the expression of $S P$ decreased 7.5 times in BOSS- $\beta$ overexpression lines, while overexpression of $B O S S-\alpha$ increased the expression of $S P$ gene by 8.6 times at $6^{\text {th }}$ leaf stage. (B) Relative expression dynamics of $S F T$ in stem apical at the different stages of seedling development.

Fig. 4. Proposed model of BOSS function as a determinant of FIN and regulating tomato

flowering. Different transcripts of BOSS regulates tomato flowering by affecting the expression level of $S P$ at seedling stage. When the transcripts of BOSS- $\alpha$ were more than BOSS- $\beta$, it can promote the expression of $S P$ and inhibit flowering. On the contrary, the expression of $S P$ was reduced when the transcripts of BOSS- $\alpha$ were more than $B O S S-\beta$, leading to the later flowering.

\section{SUPPORTING INFORMATION}

Fig. S1. Characterization of BOSS. (A) BOSS gene diagram, including 5'UTR, CDS and 3'UTR.

(B) Phylogenetic tree of BOSS and its homologous genes in different species. (C) The transcript levels of BOSS in different tomato organs: Ro, root; FB, Flower bud; Le, leaf; Fr, Fruit; St, stem; 
Fl, flower; Se, Seed.

Fig. S2. The relative expression of $B O S S$ in the stem apices of 23 selected accessions (12 SNP1 ${ }^{\text {AA }}$ accessions and 11 SNP1 $^{\text {TT }}$ accessions). Significant difference was calculated by $t$-test: $* * P<0.01$

Fig. S3. The influence of different reverse transcription methods on the detection accuracy of RNA editing efficiency. Three reverse transcription methods were used: only random primers, only oligodT primers, and combination of the two.

Fig. S4. Effect of BOSS RNA-editing on protein interaction sites (A) and subcellular localization (B, C). (A) Prediction of changes in the protein RNA interaction sites by BOSS RNA-editing (http://pridb.gdcb.iastate.edu/RPISeq/). (B, C) Subcellular co-localization of transiently expressed $B O S S$ - $\alpha / \beta$-YFP fusion protein with nuclear membrane marker (B) and plasmid membrane marker $(\mathbf{C})$ in $N$. benthamiana leaves. Bar $=10 \mu \mathrm{m}$.

Fig. S5. Overexpression of BOSS- $\beta$ leads to determinate sympodial apices. The percentages of determinate plants in the three transgenic lines are shown in Spring 2016 (A) and Spring 2017 (B).

Fig. S6. Increasing the expression of BOSS-a does not affect tomato flowering. (A) The relative expression of BOSS in BOSS- $\alpha$ overexpression transgenic tomato lines. (B) Sequence analysis at RNA-editing site reveals $0 \%$ RNA editing efficiency of $B O S S$ - $\alpha$ overexpression versus 
$72 \%$ in $\mathrm{ZY} 3$, with three independent plants used for the analysis. Color traces: G-black, A-green, T-red, C-blue. (C) Primary inflorescences (red arrowheads) and side branches (yellow arrowheads) from ZY3 (left) and BOSS- $\alpha$ overexpression line (right) with no flowering change. (D, E) Quantification and comparison of FIN (first inflorescence node) (D) and FSIN (node between first and second inflorescence) (E) in ZY3 and BOSS- $\alpha$ overexpression lines. Scale bar $=5 \mathrm{~cm}(\mathrm{C})$. All data in the graphs represent means $\pm \mathrm{SD}(n=3) . ; * P<0.05,{ }^{* *} P<0.01$.

Fig. S7. RNA-seq analysis of differentially expressed genes in the shoot apical meristem of BOSS- $\alpha / \beta$ overexpression lines versus wild type. (A, B) Volcano maps for the differentially expressed genes in BOSS- $\beta$ overexpression line (A) and BOSS- $\alpha(\mathbf{B})$ overexpression line versus the wild type. (C) GO enrichment analysis of differentially expressed genes in BOSS- $\beta$ overexpression line versus wild type. (D-F) The relative expression of three flowering-related MADS-box transcription factors was up-regulated in BOSS- $\beta$ overexpression transgenic tomato lines.

Table S1. Genes within $50 \mathrm{~kb}$ of the SNP with high relation to FIN.

Table S2. Significantly differentially expressed genes $(\log 2(\mathrm{FC})>1$ or $<-1)$ in SAM of BOSS overexpression line (p35S:: $\beta$ and p35S:: $\alpha$ ) versus wild type.

Table S3. Primers used in this study. 


\section{REFERENCES}

Abelenda JA, Navarro C, Prat S (2014) Flowering and tuberization: a tale of two nightshades. Trends Plant Sci 19: 115-122

Andres F, Coupland G (2012) The genetic basis of flowering responses to seasonal cues. Nat Rev Genet 13: $627-639$

Batistic O, Waadt R, Steinhorst L, Held K, Kudla J (2010) CBL-mediated targeting of CIPKs facilitates the decoding of calcium signals emanating from distinct cellular stores. Plant J 61: 211-222

Benne R, Van den Burg J, Brakenhoff JP, Sloof P, Van Boom JH, Tromp MC (1986) Major transcript of the frameshifted coxll gene from trypanosome mitochondria contains four nucleotides that are not encoded in the DNA. Cell 46: 819-826

Calvert A (1959) Effect of the Early Environment on the Development of Flowering in Tomato II. Light and Temperature Interactions. Journal of Horticultural Science 34: 154-162

Chen L (2013) Characterization and comparison of human nuclear and cytosolic editomes. Proc Natl Acad Sci U S A 110: E2741-2747

Degenhardt RF, Bonham-Smith PC (2008) Arabidopsis ribosomal proteins RPL23aA and RPL23aB are differentially targeted to the nucleolus and are disparately required for normal development. Plant Physiol 147: 128-142

Gerke P, Szovenyi P, Neubauer A, Lenz H, Gutmann B, McDowell R, Small I, Schallenberg-Rudinger M, Knoop V (2020) Towards a plant model for enigmatic U-to-C RNA editing: the organelle genomes, transcriptomes, editomes and candidate RNA editing factors in the hornwort Anthoceros agrestis. New Phytol 225: 1974-1992

He Y (2012) Chromatin regulation of flowering. Trends Plant Sci 17: 556-562

Imaizumi T, Kay SA (2006) Photoperiodic control of flowering: not only by coincidence. Trends Plant Sci 11: $550-558$

Jiang K, Liberatore KL, Park SJ, Alvarez JP, Lippman ZB (2013) Tomato yield heterosis is triggered by a dosage sensitivity of the florigen pathway that fine-tunes shoot architecture. PLoS Genet 9: e1004043

Jung C, Muller AE (2009) Flowering time control and applications in plant breeding. Trends Plant Sci 14: 563-573

Kardailsky I, Shukla VK, Ahn JH, Dagenais N, Christensen SK, Nguyen JT, Chory J, Harrison MJ, Weigel D (1999) Activation tagging of the floral inducer FT. Science 286: 1962-1965

Knapp S, Bohs L, Nee M, Spooner DM (2004) Solanaceae--a model for linking genomics with biodiversity. Comp Funct Genomics 5: 285-291

Kojima S, Takahashi Y, Kobayashi Y, Monna L, Sasaki T, Araki T, Yano M (2002) Hd3a, a rice ortholog of the Arabidopsis FT gene, promotes transition to flowering downstream of $\mathrm{Hd} 1$ under short-day conditions. Plant Cell Physiol 43: 1096-1105

Komiya R, Ikegami A, Tamaki S, Yokoi S, Shimamoto K (2008) Hd3a and RFT1 are essential for 
flowering in rice. Development 135: 767-774

Komiya R, Yokoi S, Shimamoto K (2009) A gene network for long-day flowering activates RFT1 encoding a mobile flowering signal in rice. Development 136: 3443-3450

Lifschitz E, Eviatar T, Rozman A, Shalit A, Goldshmidt A, Amsellem Z, Alvarez JP, Eshed Y (2006) The tomato FT ortholog triggers systemic signals that regulate growth and flowering and substitute for diverse environmental stimuli. Proc Natl Acad Sci U S A 103: 6398-6403

MacAlister CA, Park SJ, Jiang K, Marcel F, Bendahmane A, Izkovich Y, Eshed Y, Lippman ZB (2012) Synchronization of the flowering transition by the tomato TERMINATING FLOWER gene. Nat Genet 44: 1393-1398

Meng Y, Chen D, Jin Y, Mao C, Wu P, Chen M (2010) RNA editing of nuclear transcripts in Arabidopsis thaliana. BMC Genomics 11 Suppl 4: S12

Michaels SD, Amasino RM (1999) FLOWERING LOCUS C encodes a novel MADS domain protein that acts as a repressor of flowering. Plant Cell 11: 949-956

Pnueli L, Carmel-Goren L, Hareven D, Gutfinger T, Alvarez J, Ganal M, Zamir D, Lifschitz E (1998) The SELF-PRUNING gene of tomato regulates vegetative to reproductive switching of sympodial meristems and is the ortholog of CEN and TFL1. Development 125: 1979-1989

Pose D, Verhage L, Ott F, Yant L, Mathieu J, Angenent GC, Immink RG, Schmid M (2013)

Temperature-dependent regulation of flowering by antagonistic FLM variants. Nature 503: 414-417

Rajasekhar VK, Mulligan RM (1993) RNA Editing in Plant Mitochondria: [alpha]-Phosphate Is Retained during C-to-U Conversion in mRNAs. Plant Cell 5: 1843-1852

Searle I, He Y, Turck F, Vincent C, Fornara F, Krober S, Amasino RA, Coupland G (2006) The transcription factor FLC confers a flowering response to vernalization by repressing meristem competence and systemic signaling in Arabidopsis. Genes Dev 20: 898-912

Small ID, Schallenberg-Rudinger M, Takenaka M, Mireau H, Ostersetzer-Biran O (2020) Plant organellar RNA editing: what 30 years of research has revealed. Plant J 101: 1040-1056

Song YH, Ito S, Imaizumi T (2013) Flowering time regulation: photoperiod- and temperature-sensing in leaves. Trends Plant Sci 18: 575-583

Soyk S, Muller NA, Park SJ, Schmalenbach I, Jiang K, Hayama R, Zhang L, Van Eck J, Jimenez-Gomez JM, Lippman ZB (2017) Variation in the flowering gene SELF PRUNING 5 G promotes day-neutrality and early yield in tomato. Nat Genet 49: 162-168

Srikanth A, Schmid M (2011) Regulation of flowering time: all roads lead to Rome. Cell Mol Life Sci 68: 2013-2037

Suarez-Lopez P, Wheatley K, Robson F, Onouchi H, Valverde F, Coupland G (2001) CONSTANS mediates between the circadian clock and the control of flowering in Arabidopsis. Nature 410: $1116-1120$

Tang W, Luo C (2018) Molecular and Functional Diversity of RNA Editing in Plant Mitochondria. Mol Biotechnol 60: 935-945

Teo ZW, Song S, Wang YQ, Liu J, Yu H (2014) New insights into the regulation of inflorescence architecture. Trends Plant Sci 19: 158-165

Tiwari SB, Shen Y, Chang HC, Hou Y, Harris A, Ma SF, McPartland M, Hymus GJ, Adam L, Marion C, Belachew A, Repetti PP, Reuber TL, Ratcliffe OJ (2010) The flowering time regulator CONSTANS is recruited to the FLOWERING LOCUS T promoter via a unique cis-element. New Phytol 187: 57-66 
Valverde F, Mouradov A, Soppe W, Ravenscroft D, Samach A, Coupland G (2004) Photoreceptor regulation of CONSTANS protein in photoperiodic flowering. Science 303: 1003-1006

Wang Y, Gu Y, Gao H, Qiu L, Chang R, Chen S, He C (2016) Molecular and geographic evolutionary support for the essential role of GIGANTEAa in soybean domestication of flowering time. BMC Evol Biol 16: 79

Wang Y, Liu XY, Yang YZ, Huang J, Sun F, Lin J, Gu ZQ, Sayyed A, Xu C, Tan BC (2019) Empty Pericarp21 encodes a novel PPR-DYW protein that is required for mitochondrial RNA editing at multiple sites, complexes I and V biogenesis, and seed development in maize. PLoS Genet 15: e1008305

Yan J, Zhang Q, Yin P (2018) RNA editing machinery in plant organelles. Sci China Life Sci 61: 162-169

Yang Y, Fu D, Zhu C, He Y, Zhang H, Liu T, Li X, Wu C (2015) The RING-Finger Ubiquitin Ligase HAF1 Mediates Heading date 1 Degradation during Photoperiodic Flowering in Rice. Plant Cell 27: 2455-2468

Ye J, Liu GZ, Chen WF, Zhang FX, Li HX, Ye ZB, Zhang YY (2019) Knockdown of SINL33 accumulates ascorbate, enhances disease and oxidative stress tolerance in tomato (Solanum lycopersicum). Plant Growth Regulation 89: 49-58

Ye J, Wang X, Wang W, Yu H, Ai G, Li C, Sun P, Wang X, Li H, Ouyang B, Zhang J, Zhang Y, Giovannoni JJ, Fei Z, Ye Z (2020) Genome-wide association study reveals the genetic architecture of 27 yield-related traits in tomato. bioRxiv

Zhao X, Huang J, Chory J (2019) GUN1 interacts with MORF2 to regulate plastid RNA editing during retrograde signaling. Proc Natl Acad Sci U S A 116: 10162-10167 


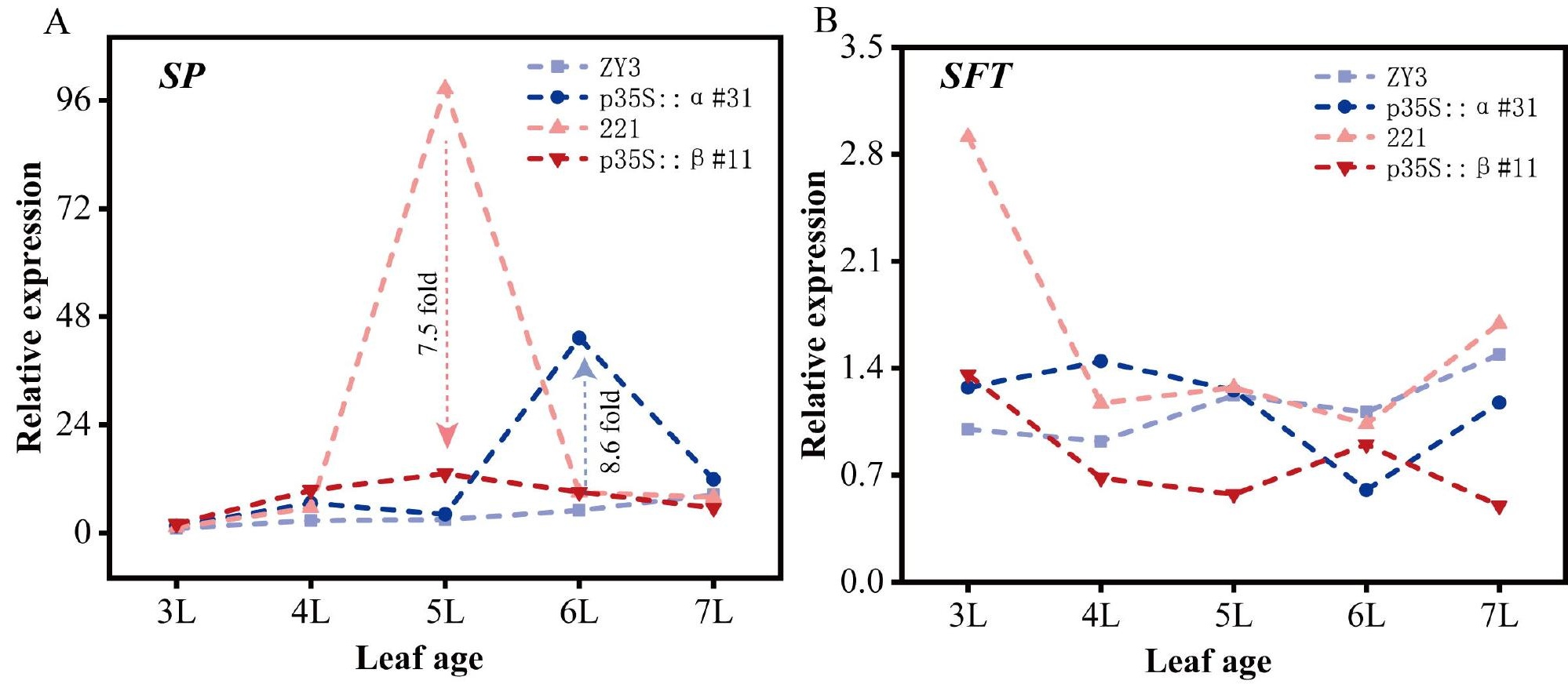




\section{Boss \\ Transcription}

bioRxiv preprint doi: https://doi.org/10.1101/2021.06.09 1202; this version posted June 9, 2021. The copyright holder for this preprin (which was not certified by peer review) is author/funder. All rights reserved. No reuse allowed without permission.

\section{(Original transcript: $\alpha$ )}

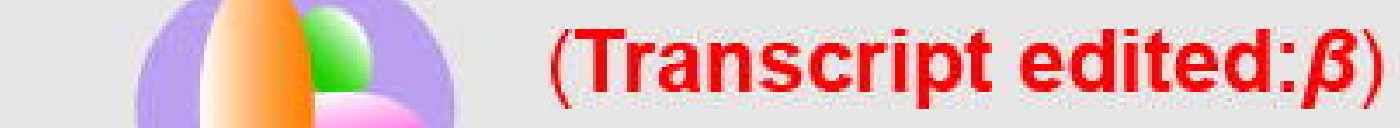

$(\alpha>>\beta)$
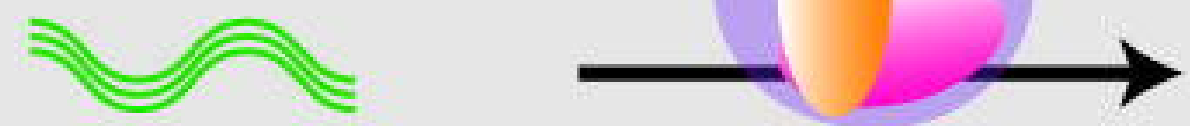

RNA editing
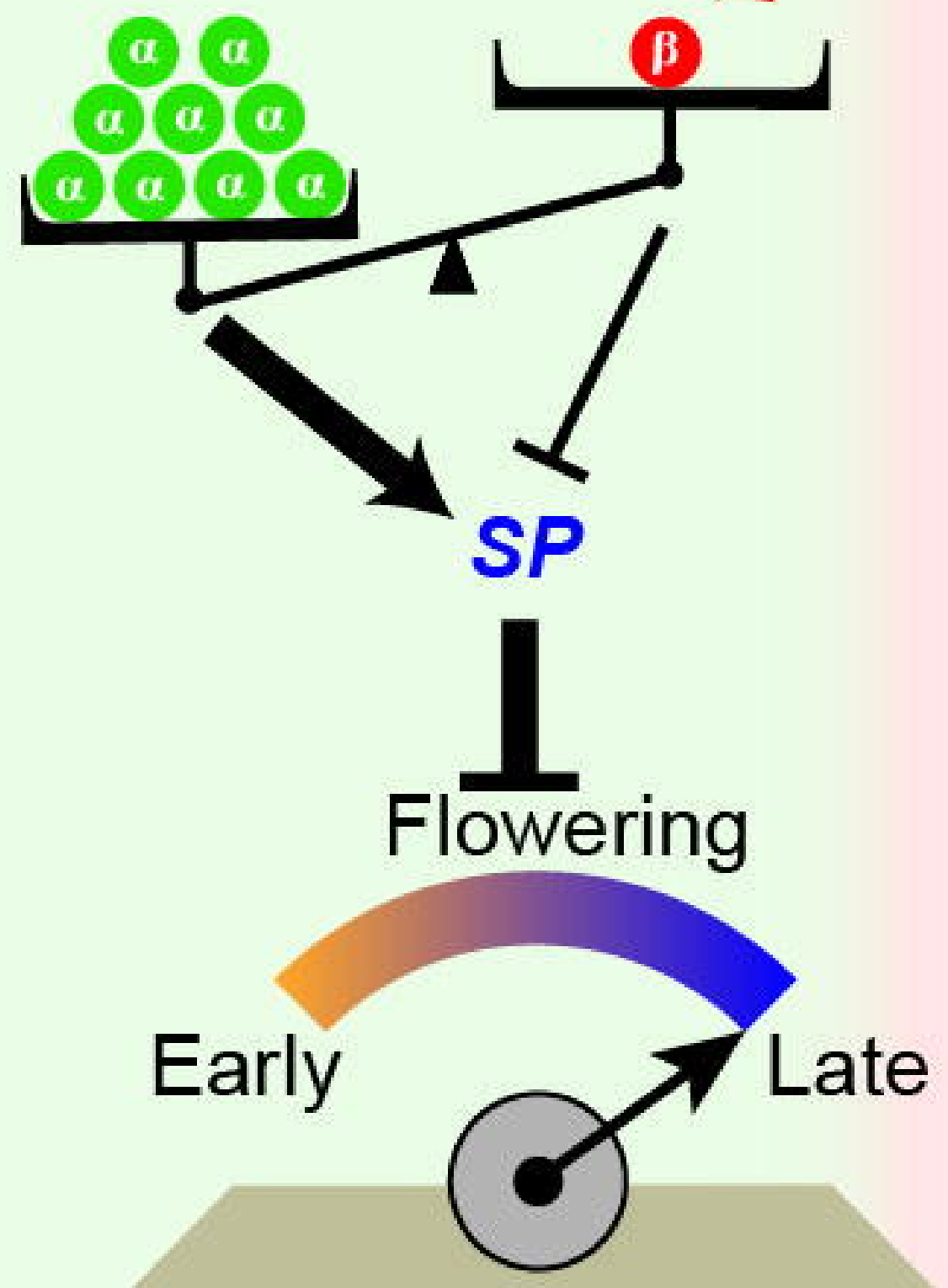

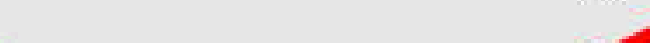

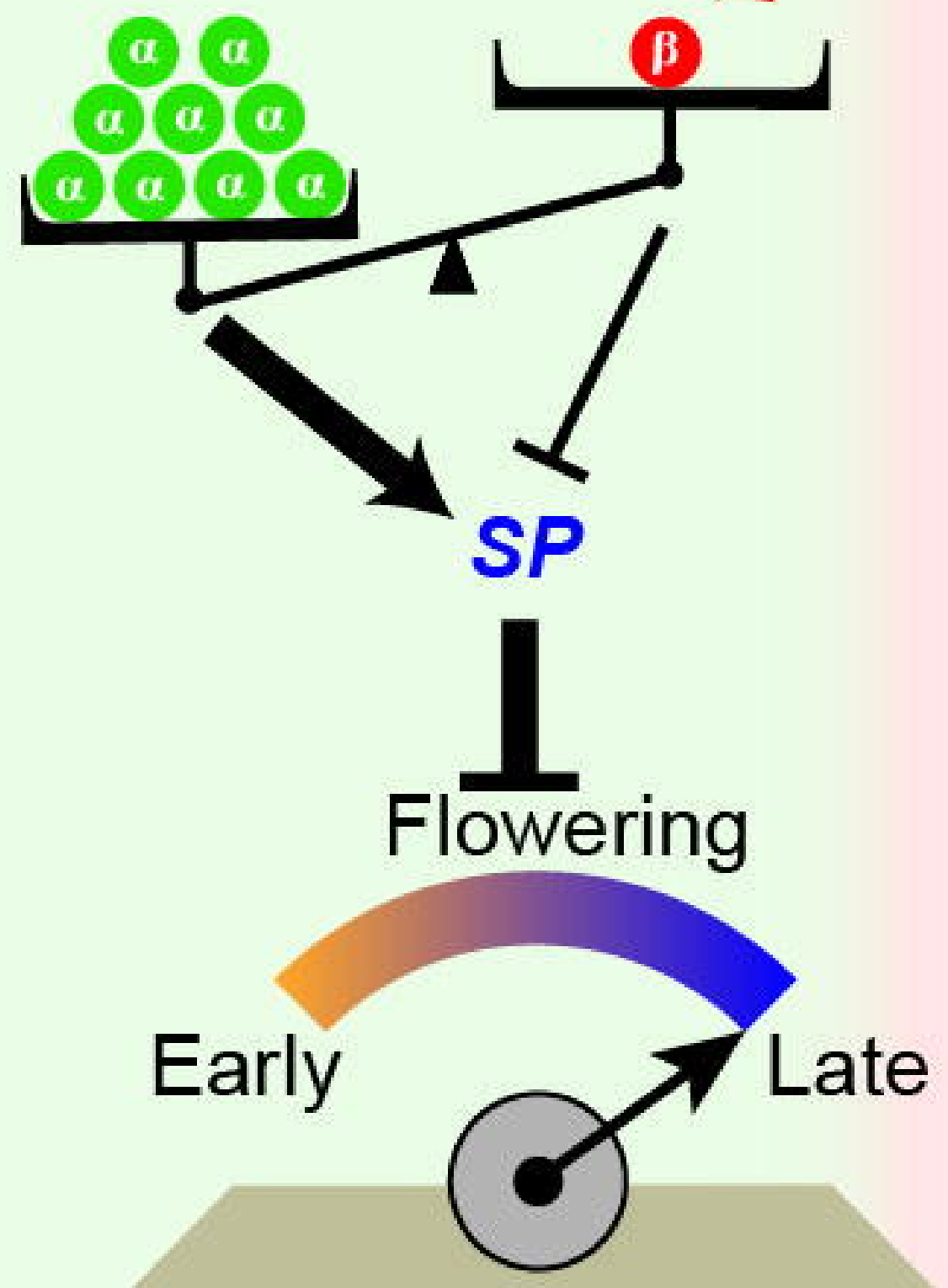
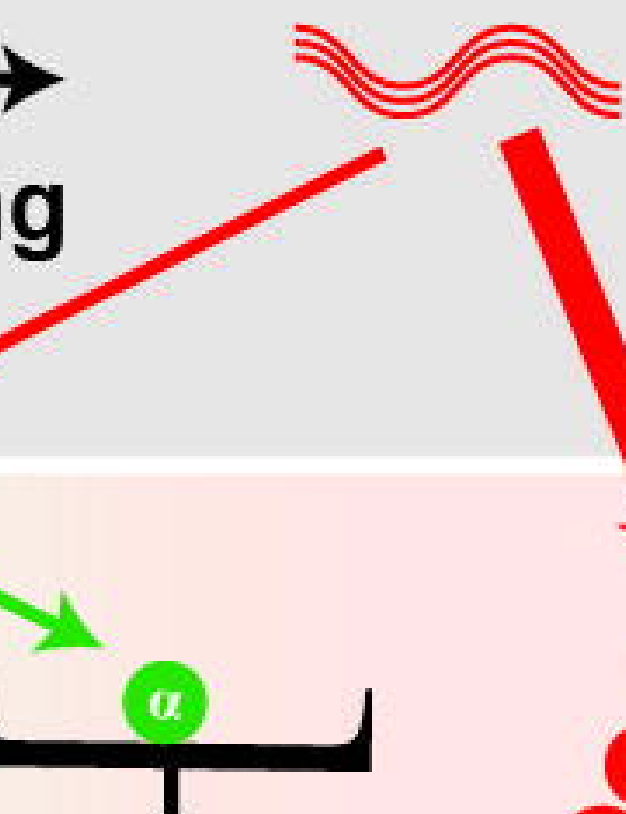

$(\alpha<<\beta)$

Early

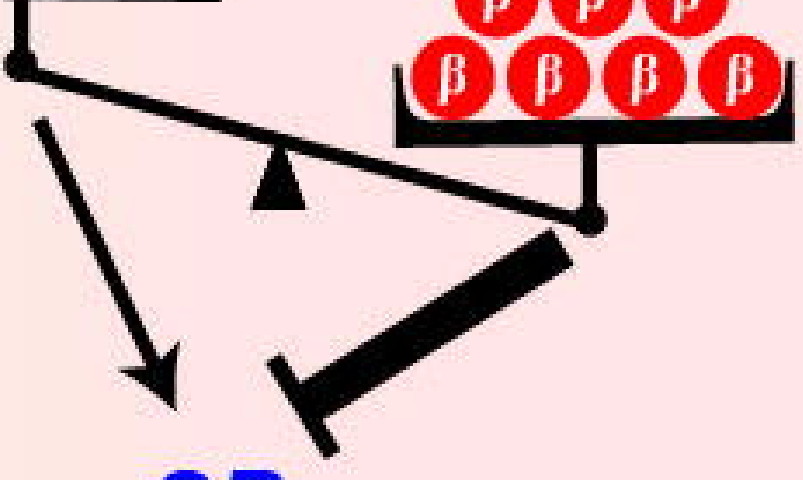

$S P$

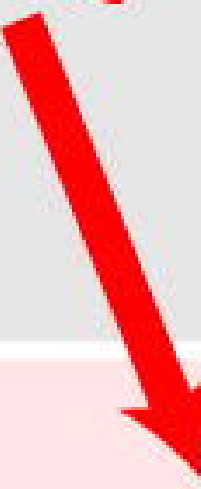

B $\beta$
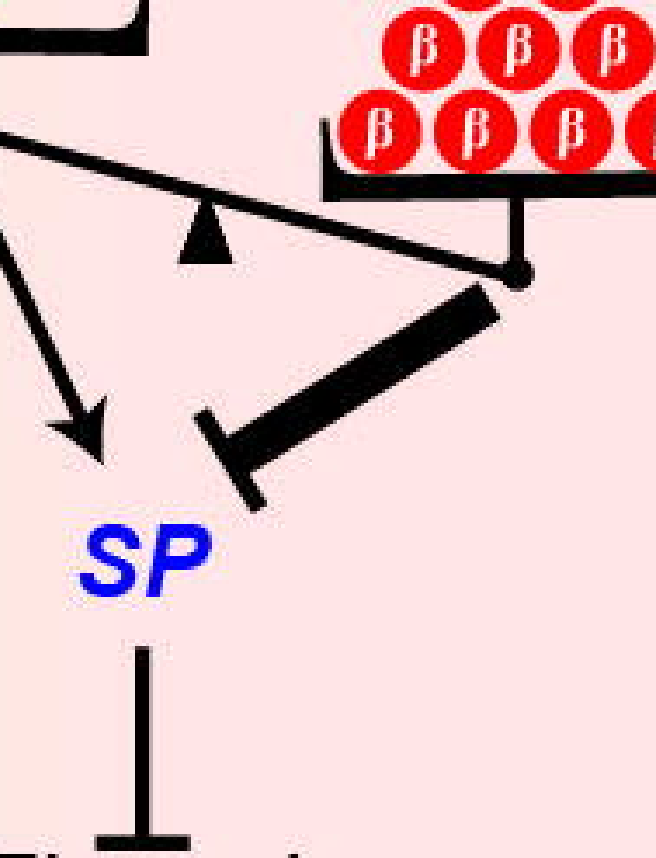

Flowering

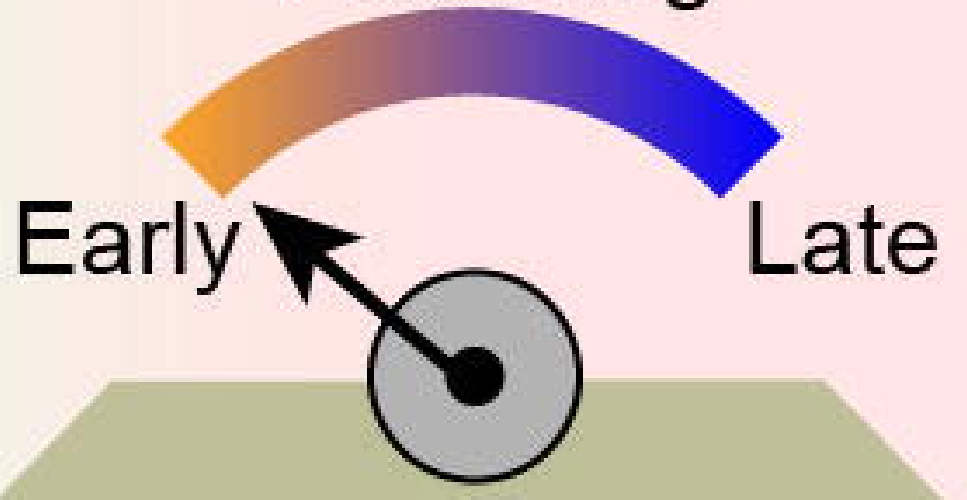

$\Rightarrow$ Strongly promoting Strongly inhibiting 